\title{
ESTIMATING EIGENVALUES OF MATRICES BY INDUCED NORMS
}

\author{
CARSTEN MiCHELS
}

Abstract. A classical result of König in terms of matrices states that for $1 \leqslant p<q \leqslant \infty$ the eigenvalues $\lambda_{1}(A), \ldots, \lambda_{n}(A)$ of an $n \times n$ square matrix $A$ satisfy $\max _{k} k^{\frac{1}{p}-\frac{1}{q}}\left|\lambda_{k}(A)\right| \leqslant C_{q, p}\|A\|_{q, p}$ for some absolute constant $C_{q, p}>0$ not depending on the matrix $A$, where $\|A\|_{q, p}$ denotes the norm of $A$ viewed as an operator from $\ell_{q}^{n}$ into $\ell_{p}^{n}$. We refine this result for $1 \leqslant p<q \leqslant 2$ by means of interpolation of Banach spaces.

Mathematics subject classification (2010): Primary: 15A60, secondary: 15A42, 65F35, 46B70, 47B06, 47B10.

Keywords and phrases: Eigenvalues, singular values, induced matrix norm, inequalities, interpolation of Banach spaces, absolutely summing norms.

\section{REFERENCES}

[1] N. ALON AND A. NAOR, Approximating the cut-norm via Grothendieck's inequality, In STOC '04: Proceedings of the thirty-sixth annual ACM symposium on Theory of computing, 72-80, New York, NY, USA, 2004. ACM.

[2] G. Bennett, Schur multipliers, Duke Math. J., 44 (1977), 603-639.

[3] J. Bergh AND J. LÖFSTRÖM, Interpolation spaces (Springer-Verlag, Berlin, 1978).

[4] A. Bhaskara and A. Vijayaraghavan, Approximating Matrix p-norms, Proceedings of the Twenty-Second Annual ACM-SIAM Symposium on Discrete Algorithms, San Francisco, CA, January 23-25, 2011. New York, NY: Association for Computing Machinery (ACM); Philadelphia, PA: Society for Industrial and Applied Mathematics (SIAM). 497-511.

[5] B. CARl And A. Defant, An elementary approach to an eigenvalue estimate for matrices, Positivity, 4 (2000), 131-141.

[6] A. Defant, Best constants for the norm of the complexification of operators between $L_{p}$-spaces, Proceedings of the Essen Conference, held in Essen, Germany, November 24 - 30, 1991. New York, NY: Dekker. Lect. Notes Pure Appl. Math. 150, 173-180 (1993).

[7] A. Defant And K. Floret, Tensor norms and operator ideals, North-Holland 1993.

[8] A. Defant, M. MastyŁo and C. Michels, Orlicz norm estimates for eigenvalues of matrices, Israel J. Math., 132 (2002), 45-59.

[9] A. Defant, M. MastyŁo and C. Michels, Eigenvalue estimates for operators on symmetric Banach sequence spaces, Proc. Amer. Math. Soc., 132 (2004), 513-521.

[10] A. DEFANT AND C. Michels, A complex interpolation formula for tensor products of vector-valued Banach function spaces, Arch. Math., 74 (2000), 441-451.

[11] J. Diestel, H. JARChOW AND A. TONGE, Absolutely summing operators, Cambridge Studies in Advanced Mathematics 43, 1995.

[12] B. Q. FENG, Equivalence constants for certain matrix norms, Linear Algebra Appl., 374 (2003), 247-253.

[13] R.A. Horn And C.R. Johnson, Topics in matrix analysis, Cambridge University Press, 1991.

[14] W.B. Johnson, H. KÖNIG, B. MAUREY AND J.R. RETHERFORD, Eigenvalues of p-summing and $\ell_{p}$-type operators in Banach spaces, J. Funct. Anal., 32 (1979), 353-380.

[15] H. KÖNIG, Eigenvalue distribution of compact operators, Birkhäuser, 1986.

[16] H. KÖNIG, Eigenvalues of compact operators with applications to integral operators, Linear Algebra Appl., 84 (1986), 111-122. 
[17] H. KÖNIG, Eigenvalues of operators and applications, in: Handbook of the geometry of Banach spaces. Volume 1. Amsterdam: Elsevier. 941-974 (2001).

[18] O. KouBA, On the interpolation of injective or projective tensor products of Banach spaces, J. Funct. Anal., 96 (1991), 38-61.

[19] M. Krein and D. Milman, On extreme points of regular convex sets, Studia Math., 9 (1940), $133-138$.

[20] J.L. KRIVIne, Sur la complexification des opérateurs de $L^{\infty}$ dans $L^{1}$, C. R. Acad. Sci. Paris, 284 (1977), 377-379.

[21] S. KWAPIEŃ, Some remarks on $(p, q)$-summing operators in $\ell_{p}$-spaces, Studia Math., 28 (1968), 327-337.

[22] C. Le Merdy, The Schatten space $S_{4}$ is a Q-algebra, Proc. Amer. Math. Soc., 126 (1998), $715-$ 719.

[23] C. Michels, One-sided interpolation of injective tensor products of Banach spaces, Bull. Belg. Math. Soc. Simon Stevin, 14 (2007), 531-538.

[24] C. Michels, On a formula of Le Merdy for the complex interpolation of tensor products, Ann. Funct. Anal., 1 (2010), 92-102.

[25] Y. NeSTERov, Semidefinte relaxation and nonconvex quadratic optimization, Optimization Methods and Software, 9 (1998), 141-160.

[26] B. OsIKIEWICZ AND A. Tonge, An interpolation approach to Hardy-Littlewood inequalities for norms of operators on sequence spaces, Linear Algebra Appl., 331 (2001), 1-9.

[27] D. STEINBerg, Computation of matrix norms with applications to robust optimization, Research thesis, Technion, Israel University of Technology, 2005.

[28] T. TAO, An epsilon of room, I: Real analysis. Pages from year three of a mathematical blog, Graduate Studies in Mathematics 117. Providence, RI: American Mathematical Society (AMS), 2010.

[29] N. TOMCZAK-JAEGERMANN, The modulii of smoothness and convexity and the Rademacher averages of trace classes $S_{p}(1 \leqslant p \leqslant \infty)$, Studia Math., 50 (1974), 163-182.

[30] A. TONGE, Equivalence constants for matrix norms: a problem of Goldberg, Linear Algebra Appl., 306 (2000), 1-13. 